\title{
Piezoelectric quartz crystal aptamer biosensor for detection and quantification of SARS CoV helicase protein
}

\author{
Dharmatov Rahula B. Albano ${ }^{1,3}$, Kato Shum ${ }^{2}$, Julian A. Tanner ${ }^{2}$, Ying Sing Fung ${ }^{1}$ \\ ${ }^{1}$ Department of Chemistry, The University of Hong Kong, Pokfulam Road, Hong Kong SAR, China \\ ${ }^{2}$ Department of Biochemistry, The University of Hong Kong, Sassoon Road, Hong Kong SAR, China \\ ${ }^{3}$ Research Center for the Natural and Applied Sciences, University of Santo Tomas, Manila \\ Philippines \\ dbalbano@ust.edu.ph
}

\begin{abstract}
:
To meet the highly demanding challenge for detecting protein biomarkers at $\mathrm{pg} / \mathrm{mL}$ level in highprotein sera sample, the coupling of paramagnetic nanoparticle technology with aptamer-PQC biosensor was attempted for developing aptamer-PQC biosensor for selective detection of SCV helicase protein produced from SARS CoV replication. The coupling of aptamer coated paramagnetic nanoparticles for sample pretreatment to aptamer-PQC biosensor has shown to detect helicase protein in one-minute assay with a detection limit of $3.5 \mathrm{ng} / \mathrm{mL}$. The aptamer-coated crystal exhibits a frequency shift linearly proportional to the concentration of SARS helicase from 0.05 to $1 \mu \mathrm{g} / \mathrm{mL}$ with a correlation coefficient of 0.9975 and a repeatability of $6.8 \%(\% \mathrm{RSD}, \mathrm{n}=3)$. After the enrichment procedure, recoveries of $102 \%$ and $119 \%$ were achieved using samples spiked with SARS helicase at concentrations of $10 \mathrm{ng} / \mathrm{mL}$ and $1.0 \mathrm{ng} / \mathrm{mL}$ respectively. It is the first report to detect for SCV helicase protein using PQC biosensor at $\mathrm{pg} / \mathrm{mL}$ level after magnetic bead enrichment.
\end{abstract}

Key words: PQC, SARS, SCV helicase, aptamer, flow injection analysis, magnetic bead 
Fast and quantitative method for the determination of SARS-CoV helicase

Due to the commercial availability of instrumentation and fast sample throughput, the flow injection analysis (FIA) system offers the most suitable method for fast and quantitative determination of protein biomarkers in biofluids. To be able to provide a fast screening method for determining analyte protein at trace level in serum, the detection mode must be sensitive, fast, and operable with label-less and direct detection. For quantitative determination of SARS-CoV helicase in human serum, a high demand has imposed on the sensitivity and selectivity of the detector. In addition, the possible contact with highly infective pathogens with serious health consequence leads to the requirement for a totally-sealed and single-used detector.

The PQC sensor provides a promising detector system as it operates by direct interaction between a target analyte in the flowing buffer and the PQC sensor surface with a specific coating. It is fast and label-less in operation with low reagent consumption and capable to handle small sample during online monitoring. The PQC crystal is cheap and the FIA setup with detector can operate under sealed condition. The sensitivity and specificity of the sensor coating towards the analyte protein are important for selection of detector.

The recently developed aptamer technology provides a suitable sensor coating for the determination of SCoV helicase protein as it was selected from a DNA library with 10 million different nucleic acids based on its strong interaction with the target protein.

Direct detection of nucleic acid interactions based on piezoelectric quartz crystal was first reported by Fawcett et al. in 1988[1], leading to a rapid development of new sensor systems based on using nucleic acid with a given sequence for sensing a specific analyte protein.

The 5'-biotin-labeled aptamer solutions of a concentration of $2 \mathrm{mmol} / \mathrm{mL}$ in PBS buffer were heated to $95 \circ \mathrm{C}$ for $3 \mathrm{~min}$, chilled on ice to place the aptamer in its correct intramolecular folding and. Subsequently, $10 \mu \mathrm{L}$ of aptamer solution was added onto crystal surface and incubated at room temperature for 1 hour after removal of unbound aptamers by rinsing 3 times with PBS buffer. To block unreacted surface groups, $10 \mu \mathrm{L}$ of a $0.025 \%$ BSA solution was applied, following by incubation at room temperature for 1 hour before rinsing 3 times with PBS buffer[2].

The usage of aptamer coated magnetic particle eliminates other proteins that may interfere with the detection of SCV helicase proteins and at the same time enrichment is carried out in order to measure beyond the detection limit of the PQC sensor. Table 1 shows the enrichment of a prepared serum sample containing $1.0 \mathrm{ng}$ and $10 \mathrm{ng}$ SCV helicase proteins $1000 \mu \mathrm{L}$ serum. The total volume of the samples before enrichment was $1000 \mu \mathrm{L}$ and it was reduced to $100 \mu \mathrm{L}$ by magnetic particles coated with NG-8 aptamer which was the same aptamer used in the PQC sensor. Results show an increase in the frequency response for both samples. The frequency response after enrichment was correlated to the concentration in the standard calibration curve as shown Figure 1. After purification of the sample by a magnetic bead, an enrichment of 10 fold was achieved. After the enrichment procedure, recoveries of $102 \%$ and $119 \%$ were achieved using samples spiked with $10 \mathrm{ng} / \mathrm{mL}$ and $1.0 \mathrm{ng} / \mathrm{mL}$ respectively with SARS helicase. It is the first report to detect for SCV helicase protein using PQC biosensor at $\mathrm{pg} / \mathrm{mL}$ level after magnetic bead enrichment.

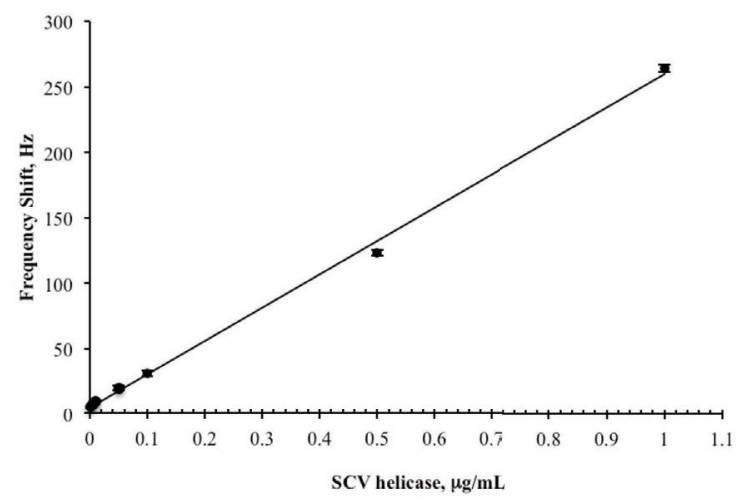

Figure 1. The working curve for using aptamer $P Q C$ sensor for SCV helicase determination. Buffer flow rate $1.0 \mathrm{~mL} / \mathrm{hr}$, sample loop volume $20 \mathrm{~mL}$, buffer = $P B S$ at $p H=7.2$. For SCV helicase from 0.01 to 1 $\mathrm{mg} / \mathrm{mL}, y=254.71 x+4.8675, R^{2}=0.9975$.

The enrichment of the SCV helicase using an aptamer bound to the magnetic bead proves to provide an effective way for sample purification. As a result, the response of the aptamer PQC sersor was increased as shown in the sensorgram of a $1 \mathrm{ng} / \mathrm{L}$ spiked serum sample in Figure 2. Based on the calibration curve, the concentration of the $1,000 \mathrm{~mL}$ serum sample spiked with $1 \mathrm{ng} / \mathrm{mL}$ SCV helicase was enriched 10 times to $100 \mathrm{~mL}$ serum sample.

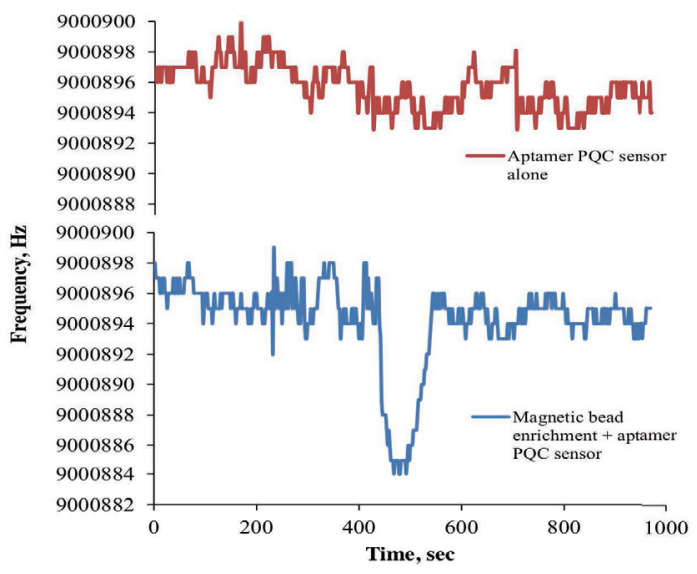


Figure 2. Aptamer PQC sensorgram of $1000 \mu \mathrm{L}$ serum sample spiked with $10 \mathrm{ng} / \mathrm{mL}$ SCV helicase. Flow rate $=1.0 \mathrm{~mL} / \mathrm{hr}$, sample loop volume $=20 \mathrm{~mL}$.

\section{References:}

[1] N.C. Fawcett, J.A. Evans, L.C. Chien, N.

Flowers, Nucleic Acid Hybridization Detected by Piezoelectric Resonance, Anal. Lett. 21 (1988) 1099-1114.

http://www.informaworld.com/10.1080/00032 718808055499

[2] M. Liss, B. Petersen, H. Wolf, E. Prohaska, An aptamer-based quartz crystal protein biosensor, Anal. Chem. 74 (2002) 44884495. doi:10.1021/ac011294p. 